\title{
How clean is clean: a new approach to assess and enhance environmental cleaning and disinfection in an acute tertiary care facility
}

\author{
Wai Khuan $\mathrm{Ng}$
}

Mafraq Hospital, United Arab Emirates

\begin{abstract}
Traditional environmental cleaning monitoring through visual assessment can identify gross lapses in practice. However, in recent years the limitations underlying this need for ongoing compliance with cleaning and disinfection policies in the patient's immediate surroundings have become widely recognised.

The value of objectively monitoring and improving environmental cleaning and disinfection in healthcare settings is becoming increasingly identified as a crucial element of strategies to mitigate the transmission of healthcare-associated infections. Mafraq Hospital has adopted a new method using an invisible fluorescent marker system to target on surfaces in patient's immediate surroundings. Evaluation of at least 30 surfaces and objects in patient rooms revealed that only $11 \%$ of targets had been cleaned. Simulation training, educational interventions, empowerment, change involvement and acknowledgment were executed, leading to a sustained improvement of $77 \%$ in both quarter 2 and 3 of 2013 in cleaning and disinfecting of all surfaces and objects.
\end{abstract}

\section{Problem}

Patients with pathogens (eg, methicillin-resistant Staphylococcus aureus, vancomycin-resistant Enterococcus, Acinetobacter) frequently contaminate environmental surfaces in their immediate surroundings. These organisms can remain viable in the environment for weeks and months. Items in patients' immediate vicinity are frequently contaminated, such as bed rails, bed linen, overbed tables, blood pressure cuffs, TV remote control, nurse call buttons, etc.[1,2,3]

\section{Background}

Contaminated environmental surfaces contribute to pathogens transmission, and it serves as a source from which healthcare workers contaminate their hands and gloves.[4] Contaminated medical equipment that has direct contact with the patient can serve as a source of transmission.[3, 4] Patients admitted to a room formerly occupied by a patient with a multidrug-resistant organism (MDRO) are at increased risk of acquiring the organism, suggesting inadequate terminal cleaning of rooms or organism acquisition directly from the contaminated surfaces, or from contaminated hands of health care workers. $[3,4,5,10,11]$ Cleaning practices are frequently suboptimal and daily cleaning of surfaces near patients is often performed poorly. In addition, terminal cleaning of rooms after patient discharge is often inadequate.[4, 5, 11] Carling et al (2013) found that only $47 \%$ of surfaces targeted for terminal cleaning had been cleaned.[6]

The contributing factors could be that environmental service personnel and nurses often do not agree on who should clean what. $[6,7]$ Perhaps the environmental service personnel do not understand which detergent or disinfectant to use, what concentration should be used, or how often to change cleaning cloths or mop heads. [7] Other factors leading to this deficit could also be demands for fast room turnaround times, staff shortages, frequent turnover of personnel, and lack of a standardised mechanism to monitor the effectiveness of cleaning and disinfection practices.[7]

There is increasing recognition of the contribution and role of the environment in the transmission of healthcare-associated infections. Over the past decade, numerous studies have shown that approximately $30 \%$ to $60 \%$ of environmental surfaces in the patient zone of individuals colonised or infected with C. difficile, MDRO (eg, VRE, MRSA) are contaminated with these microorganisms.[2, 3, 4, $5,10,11,19,20]$ Although less widely studied, multiple reports have identified similar rates of contamination with Acinetobacter baumanii in patient rooms occupied by colonised and infected cases.[2, 3, 13] Studies have shown that improved cleaning and disinfection of environmental surfaces can reduce transmission of pathogens such as C. difficile, MDRO (eg, VRE, MRSA).[2, 3, 4, 5, $14,19,20]$ Therefore, the healthcare facilities have started to educate the environmental service personnel, develop check list to ensure environmental surfaces have been wiped or mopped, and develop policies regarding which patient-care equipment and environmental surfaces are to be cleaned by environmental service personnel and by nurses. $[6,7,15,16]$

Although environmental cleaning and disinfecting practices have become a cornerstone of patient care, assessment of actual compliance at Mafraq Hospital was not performed.

\section{Baseline measurement}

The infection preventionists conducted a literature review to analyse the evidence-based practices that led to improved environment 
cleaning. Four methods have been identified, which are: visual inspection, environmental culturing, adenosine triphosphate (ATP) bioluminescence assay systems, and fluorescent marker system. [6, $7,8,9,18,21,22,23,24,25]$

Visual inspection is a simple method but does not provide a reliable assessment of cleanliness. Environmental culturing is relatively simple to detect presence of pathogens but it is more costly and the results are not available until 48 hours later. Although ATP bioluminescence assay systems do provide quantitative measure of cleanliness and quick results, it is also an expensive method and it requires special equipment.[6, 7, 8, 9, 21, 23] As for fluorescent marker system, it is inexpensive when comparing with environmental culturing and ATP bioluminescence assay systems, and it only requires minimal equipment. However, it requires marking surfaces before cleaning, and checking them after cleaning. $[6,7,8,9]$

Biosimulators and visual training is an important new approach for learning in the health care setting; it allows health care workers to see the impact of disease transmission compared to traditional didactic education.[9] ATP bioluminescence assay systems and fluorescent marker system were discussed during the infection prevention and control committee meeting to conclude on method which Mafraq Hospital will adopt to evaluate the cleaning and disinfection techniques.

After cost consideration, a fluorescent marker system was adopted by the committee. The infection prevention and control committee members were also briefed that at least 10 high-touch surfaces or objects in patient rooms will be marked with fluorescent powder by the secret auditor before cleaning, and marks moistened by disinfectant could be removed by wiping surface for 5 seconds with light pressure. The evaluation will be performed in patient rooms, following discharge of patients, before being occupied by next patient. Target surfaces will be evaluated using a portable ultraviolet light to see if the marker had been wiped off.

See supplementary file: ds3770.docx - "Fluorescent Marker System"

\section{Design}

In post-natal unit and in operating theatres, a total of 30 high touch surfaces (eg, door handle, alcohol-based hand dispenser, water faucet, overhead table, telephone keypad, etc) were evaluated. The surfaces that were cleaned of the fluorescent powder are considered "clean" and counted as compliance. In calculating the compliance rate, the numerator is the number of clean surfaces. The denominator is the total number of surfaces evaluated. Thus, the initial compliance rate was $11 \%$.

When considering the underlying cause of this poor compliance rate $(11 \%)$, it became clear that the environment and equipment were not appropriately disinfected. An intervention needed to be designed to be able to help improve this. A number of ideas were considered for how to achieve this, including developing policies, educating the environmental service personnel on proper concentration of disinfectant dilution as well as not reusing the same cleaning cloth for surface or equipment disinfection, however neither of these ideas was deemed practical nor sustainable.[16, 17]

The agreed intervention was that the environmental service team, post-natal unit manager, OT unit manager, infection prevention, and control link practitioners (ie, nurses selected from individual unit) were trained in using the fluorescent marker system to evaluate the cleaning and disinfection techniques in their respective areas. Scheduled evaluation was performed by the infection preventionists and prompt feedback was provided. The units or departments with top compliance were acknowledged by the senior management. This idea was felt to be sustainable for the following reasons:

i. The environmental service team will use the system to train their staff in proper cleaning and disinfection techniques, as practices tend to be sustainable with biosimulators and visual training

ii. The unit or department managers and link practitioners evaluate the cleaning and disinfection techniques in their own areas; we believe that change tends to happen when the process is owned by the stakeholders

iii. The units or departments always want to prove that they are the best and be acknowledged.

\section{Strategy}

The cleaning and disinfection technique evaluation process provided highly positive results in favor of objectively monitoring and improving environmental cleaning and disinfection practices at Mafraq Hospital using fluorescent marker system and thus did not necessitate the need for the 3rd PDSA cycle.

PDSA cycle 1: At least 30 high-touch surfaces or objects were marked with fluorescent powder by the secret auditor before cleaning. The evaluation was performed in patient rooms following the discharge of patients but before being occupied by next patient. Target surfaces were evaluated using a portable ultraviolet light to see if the mark had been wiped off. The compliance rate was only $11 \%$ in 1 st quarter 2013. Factors led to the non-compliance were analysed, and these were: lack of supervision for cleaning procedure, no standardisation in allocating time for cleaning procedure, knowledge deficit about the concentration and preparation of surface disinfectant, quick cleaning on demand during high turnover of patients, the same disposable cleaning cloth for clean and contaminated surfaces or equipment, elbow grease (manual friction) was not used during cleaning, equipment missed to be cleaned, staff shortage, frequent turnover of environmental personnel and lack of standardised mechanism to monitor the effectiveness of cleaning and disinfection practice.

Based on the findings, the environmental service team enhanced the cleaning and disinfection training program. The nursing unit managers and infection prevention and control link practitioners were trained on using the fluorescent marker system to evaluate the cleaning and disinfection techniques in their respective areas. The 
cleaning and disinfection technique evaluation was adopted as an internal key performance measure by OT unit manager. Scheduled evaluation was performed by the infection preventionists and prompt feedback was provided.

PDSA cycle 2: When the practices were reevaluated in 2nd quarter 2013, the cleaning and disinfection technique compliance has increased to $76.9 \%$, after at least 30 surfaces of environment and equipment were evaluated. The results were shared with the key stakeholders. The test proved that the transmission of healthcareassociated pathogens can be mitigated through objectively monitoring and improving environmental cleaning and disinfection practices in healthcare settings. Fluorescent dye marker system was rolled out to the whole Mafraq Hospital, in addition to the regular audits and ongoing education. The units or departments with top compliance were acknowledged by the senior management.

See supplementary file: ds4073.docx - "PDSA Cycle 1 \& 2 How Clean is Clean"

\section{Post-measurement}

When the system was initiated in February 2013, the cleaning and disinfection technique compliance rate was only $11 \%$ in the 1 st quarter 2013. When the practices were reevaluated in the 2nd quarter 2013, the cleaning and disinfection technique compliance had increased to $76.9 \%$ and sustained at $77.3 \%$ in the 3 rd quarter 2013. However, the cleaning and disinfection technique compliance decreased to $50 \%$ in 4 th quarter 2013 as the link practitioners and environmental service team were not empowered and given the ownership to closely monitor the practice in their respective areas. Once the stakeholders were empowered, ownership were enhanced and top performers were acknowledged by the senior management team, the compliance increased to $75 \%$ in 1 st quarter 2014.

"Organising the change by organising the power - change will happen only by getting people involved."

See supplementary file: ds3783.pdf - "How Clean is Clean Post Measurement"

\section{Lessons and limitations}

Traditional environmental cleaning monitoring through visual assessment can identify gross lapses in practice. However, in recent years the limitations underlying this need for ongoing compliance with cleaning and disinfection policies in the patient's immediate surroundings have become widely recognised.[7, 8, 15, $17,22]$ Although the Centers for Disease Control and Prevention (CDC) recommends that healthcare facilities ensure compliance by environmental service personnel with cleaning and disinfection procedures, our results objectively demonstrated an opportunity to significantly improve the cleaning practices of patient's immediate surroundings.[14]
In spite of the fact that this study objectively documented improved environmental cleaning and disinfection techniques in the patient care areas of Mafraq Hospital, an association between improved compliance with cleaning practices and healthcare-associated infection or cross-transmission was not able to be evaluated.

\section{Conclusion}

Given the widespread concerns regarding the ability of current strategies, including hand hygiene and antimicrobial stewardship programs intended to decrease prevalence of multidrug-resistant organisms in the healthcare settings, and in light of the fact that numerous studies have clearly indicated that enhanced cleaning and disinfection techniques significantly decrease environmental contamination with a range of healthcare-associated organisms which has led to decreased healthcare-associated infections or the transmission of healthcare-associated organisms, it is conspicuous to optimise environmental cleaning and disinfecting activities in healthcare facilities.[20]

We believe that the fluorescent marker intervention described in this article has the potential to quantitatively assess cleaning and disinfecting practices. By combining this assessment with educational activities that integrate prompt and direct objective feedback for the environmental service team, cleaning and disinfecting practices can be improved and these gains can be sustained.

\section{References}

1. Luick L, Thompson PA, Loock MH, Vetter SL, Cook J, Guerrero DM. Diagnostic assessment of different environmental cleaning methods. Am J Infect Control 2013; 41:751-2.

2. Chang S, Sethi AK, Eckstein BC, Stiefel U, Cadnum JL, Donskey CJ. Skin and environmental contamination with methicillin-resistant Staphylococcus aureus in patients identified clinically versus through active surveillance. Clin Infect Dis 2009. 48:1423-8.

3. Bhalla A, Pultz NJ, Gries DM, Ray AJ, Eckstein EC, Aron $D C$, et al. Acquisition of nosocomial pathogens on hands after contact with environmental surfaces near hospitalized patients. Infect Control Hosp Epidemiol 2004. 25:164-7.

4. Guerrero DM, Nerandzic MM, Jury LA, Jinno S, Chang S, Donskey CJ. Acquisition of spores on gloved hands after contact with the skin of patients with Clostridium difficile infection and with environmental surfaces in their rooms. Am J Infect Control 2012;40:556-8.

5. Sethi AK, Al-Nassir WN, Nerandzic MM, Bobulsky GS, Donskey CJ. Persistence of skin contamination and environmental shedding of Clostridium difficile during and after treatment of Clostridium difficile infection. Infect Control Hosp Epidemiol 2010. 31:21-7.

6. Carling PC, Briggs, JL, Perkins J, Highlander D. Improved cleaning of patient rooms using a new targeting method. Clin Infect Dis 2006. 42(3):385-88.

7. Thomas C. Environmental Services Performance 
Improvement in the ICU Using a Cleaning Checklist, Educational Video, and Fluorescent Marking Solution with Rapid-Cycle Feedback. Am J Infect Control 2011. 39:176.

8. Yuen TK, Lin LM, Choon LE, Yan GY, Sun CC, Chuo Ying $\mathrm{CY}$, Hong KY. New approaches to enhance environmental cleaning in an acute tertiary hospital. BMC Proceedings 2011. (6):302.

9. Po JL, Burke R, Sulis C, Carling PC. Dangerous cows: An analysis of disinfection cleaning of computer keyboards on wheels. Am J Infect Control 2009. 37:778-80.

10. Hayden MK, Blom DW, Lyle EA, Moore CG, Weinstein RA. Risk of hand or glove contamination after contact with patients colonized with vancomycin-resistant enterococcus or the colonized patients environment. Infect Control Hosp Epidemiol 2008. 29:149-54.

11. Fellowes C, Kerstein R, Clark J, Azadian BS. MRSA on tourniquets and keyboards. J Hosp Infect 2006. 64:86-8.

12. Rutala WA, White MS, Gergen MF,Weber DJ. Bacterial contamination of keyboards: efficacy and functional impact of disinfectants. Infect Control Hosp Epidemiol 2006. 27:372-7.

13. Bures S, Fishbain JT, Uyehara CF, Parker JM, Berg BW. Computer keyboards and faucet handles as reservoirs of nosocomial pathogens in the intensive care unit. Am J Infect Control 2000. 28:465-71

14. Sehulster L, Chinn RYW. Guidelines for Environmental Infection Control in Healthcare Facilities: recommendations of the CDC and the Healthcare Infection Control Advisory Committee. MMWR 2003. 52:1-42.

15. Carling $P$. Methods for assessing the adequacy of practice and improving room disinfection. Am J Infect Control 2013. 41:20-25.

16. Dancer SJ. Control of transmission of infection in hospitals requires more than clean hands. Infect Control Hosp Epidemiol 2010. 31:958-60.

17. Carling PC, Bartley JM. Evaluating hygienic cleaning in healthcare settings: what you do not know can harm your patients. Am J Infect Control 2010. 38:S41-50.

18. Carling PC, Briggs J, Hylander D, Perkins J. Evaluation of patient area cleaning in 3 hospitals using a novel targeting methodology. Am J Infect Control 2006. 34:513-9.

19. Morgan DJ, Liang SY, Smith CL. Frequent multidrugresistant Acinetobacter baumannii contamination of gloves, gowns, and hands of healthcare workers. Infect Control Hosp Epidemiol 2012. 31:716-21.

20. Nseir S, Blazejewski C, Lubret R, Wallet F, Courcol R, Durocher A. Risk of acquiring multi-drug resistant gramnegative bacilli from prior room occupants in the intensive care unit. Clin Microbiol Infect 2011. 17:1201-8.

21. Miller BA, Sexton $\mathrm{OH}$. Shedding light on new methods to improve hospital cleanliness: the use of ultraviolet monitors as surrogate markers for bacterial contamination. Crit Care Med 2010. 38:1212-4.

22. Dancer SJ. Hospital cleaning in the 21 st century. Eur J Clin Microbiol Infect Dis 2011. 30:1473-81.

23. Sitzlar B, Deshpande A, Fertelli D, Kundrapu S, Sethi AK, Donskey CJ. An environmental disinfection odyssey: evaluation of sequential interventions to improve disinfection of clostridium difficile isolation rooms. Infect Control Hosp Epidemiol 2013. 34(5):459-65.

24. Gordon L, Bruce N, Suh KN, Roth V. Evaluating and operationalizing an environmental auditing program: A pilot study. Am J Infect Control 2014. 42:702-7.

25. Beam EL, Gibbs SG, Boulter KC, Beckerdite ME, Smith PW. A method for evaluating health care workers' personal protective equipment technique. Am J Infect Control 2011. 39:415-20.

\section{Declaration of interests}

Nothing to declare.

\section{Acknowledgements}

Co-authors:

Prameela Maniamma, Reham Jafer, Fathia Jama Osman, Jancy Varghese, Helge Springhorn, Asim Malik. 